\title{
A GIANT PARAUMBILICAL ABDOMINAL MASS
}

Musa Balowa (MD5 2006/2007)

\section{INTRODUCTION:}

Subcutaneous fibrolipoma is a rare neoplasm that is defined as a subtype of lipoma'. Lipomas are the most common benign mesenchymal tumor and are composed of mature fat cells. Benign fatty tumours can arise in any location in which fat is present. They are found in the subcutaneous tissues (neck, back, and proximal extremities are affected most commonly by lipomas ${ }^{3}$ ) and, less commonly, in internal organs. They usually arise in early adulthood and are rare in children and infants. Most patients affected by such tumours are in their fifth or sixth decade of life. Lipomas may be single or multiple. Solitary lipomas are seen predominately in women while multiple lipomas occur more frequently in men².

\section{CASE REPORT:}

A. C. M. fifty years old female from Dodoma, she is three years post menopausal and attained menarche at 12 years.

She was referred from Ocean Road Cancer Institute with an ulcerated abdominal swelling. The swelling grew rapidly and within two months it became a large painless mass that ulcerated over two months, it became painful with watery, whitish yellow foul smelling discharge. The ulcer was not bleeding and there was no itching. She was well until four months back when she noticed a small pimple on the scar of previous surgery. Seven years back she had similar swelling at the same site and was excised at Itigi. Other parts of the body were free of similar lesions. A positive history of smoking and consuming local brew for the past twenty years was retrieved.

The swelling had no association with fever, weight loss, cough or difficult in breathing, back pain, bone pains, joint stiffness, joint deformity loss of limb movements and numbness nor was there a history of breast lump, pain or discharge.

She is a housewife married to a peasant with no family history of cancer (e.g. breast cancer), diabetes mellitus, hypertension or tuberculosis.

On examination a large ovoid left para umbilical mass, $12 \mathrm{~cm}$ by $14 \mathrm{~cm}$ in size stretching the skin. Not reducible and no impulse on cough. The swelling was not warm. It was non tender, smooth, firm to hard in consistency with soft compressible edges. It was not fluctuant; the base was fixed to the abdominal wall. There was no bruit and Trans-illumination was negative. Two small nodules of similar consistency about $0.5 \mathrm{~cm}$ by $0.5 \mathrm{~cm}$ adjoining the base were visibly noticed. There was a $5 \mathrm{~cm}$ by $8 \mathrm{~cm}$ ovoid with slopping edges ulcer on the upper surface of the swelling. The floor was covered with smooth tiny granulation tissue which bled easily about $3 \mathrm{~mm}$ deep into the swelling, serous foul smelling discharge was released, the base was non tender and was not indurated and fixed to the swelling, the surrounding skin was tense and glossy.

Inguinal and axillary lymph nodes were not palpable. Femoral and popliteal pulses were symmetrically palpable.

Superficial abdominal reflexes were sluggish and could not be elicited on the left side, but the knee, ankle and planter (flexor) reflexes were normal. Incision biopsy revealed chronic inflammation, granulation tissue due to many plasma cells, neutrophils, granulocytes, vascular proliferation, congestion, oedema and granulation tissue with no features of Dermatofibrosarcoma protuberans. Haemoglobin level was $12.3 \mathrm{~g} / \mathrm{dL}$, \& chest X-ray was clear. Wide local excision was done and subsequent histology revealed fibrolipoma and the tumour margins reported free of tumour cells.

\section{DISCUSSION:}

Most patients affected by such tumours are in their fifth or sixth decade of life which was demonstrated in this case. Solitary lipomas are seen predominately in women which was the presentation in this case while multiple lipomas occur more frequently in men ${ }^{2}$. This is in contrary to the case report of Mazzocchi et al who reported that multiple lipomas are more common in women; many are seen in a familial setting ${ }^{3}$. Usually it 
is a long standing tumour but the exact duration may not be discovered as it may remain unnoticed for months ${ }^{4}$. However in the present history the duration may be exactly short as the patient might be too alert of the condition following the history of similar tumour. Neck, back, and proximal extremities are affected most commonly by lipomas ${ }^{3}$, occurrence on anterior abdomen is unusual. Lipomas present as subcutaneous nodules of 2-10 cm, often are lobulated, rubbery in consistency, skin overlying the lesion is normal and is not connected to the tumor. The clinical features found in this case are quite different from the above presentation probably due to delay in care seeking hence stretching and disruption of the skin overlying the tumor as it has no capsule with necrosis. Recurrence of lipofibroma is not documented however it might follow inadequate excision of the primary tumour. It was found that when a tumour was (a) larger than $5 \mathrm{~cm}$, irrespective of depth and location, (b) located in the thigh, irrespective of depth and size, or (c) deep, irrespective of location and size, it was more likely to be a sarcoma ${ }^{5}$. This presentation reflected of including liposarcoma in the differential diagnosis thigh was not affected by the tumour. When very close to vital structures, giant lipomas may, on account of their excessive size, cause functional limitations, such as lymphedema, pain or nerve compression syndromes ${ }^{5}$.

This was not observed in this case due to the lesion being away from the inguinal region. In the investigation of the patient with lipomas fine-needle aspiration cytology (FNAC) is indicated when the diagnosis of liposarcoma is suspected. In this case incisional biopsy was done instead of fine needle aspiration cytology which revealed non specific results. This is probably due to superficial incision into the ulcer or on the skin surrounding the ulcer. Also CT scanning is indicated in suspected liposarcoma as it is useful for distinguishing between lipomas and liposarcomas². Angiography may demonstrate tumor malignancy on the basis of prominent vascularity; thus, angiography may be of value in planning surgical resection. Chest radiography may be used as an initial screening for pulmonary metastases; however, the definitive test for detection of pulmonary metastases is chest $\mathrm{CT} \mathrm{scan}^{2}$. In this case angiography was not done. However chest radiograph did not show evidence of pulmonary metastasis hence good prognosis.

Lipomas are treated surgically by simple surgical excision, liposuction and squeeze technique. None of the above treatment modality was used however wide local excision was done through an ovoid incision a highly vascularised tumour limited to the skin and subcutaneous tissues and normal rectus sheath was found, tumor was entirely excised, and a part of the specimen was taken for histology. The finding of fibrolipoma and having tumour free margins suggests eradication of the tumour hence prevention of recurrence.

Due to rarity of fibrolipoma there is limited literature hence age was the only predisposing factor documented so far and certain literatures have managed to define the condition.

\section{RECOMMENDATIONS:}

It is recommended that all patients with suspected giant fibrolipomas to undergo fine needle aspiration cytology to be followed later with local wide excision with part of the tumour and marginal tissues to be sent for histology to rule out malignancy and decrease rate of recurrence.

\section{REFFERENCES:}

1. Kajihara M, Sugawara Y, Sakayama K, Abe Y, Miki H, Mochizuki T. Subcutaneous fibrolipoma in the back. Radiat Med. 2006 Aug;24(7):520-4.

2. Moraru RA (2006). Dermatology: benign neoplasms: Lipomas. www.eMedicine.com. (Accessed on June 10, 2007)

3. Mazzocchi M, Onesti MG, Pasquini P, La Porta R, Innocenzi D, Scuderi N. Giant fibrolipoma in the leg--a case report. Anticancer Res. 2006 SepOct;26(5B):3649-54

4. Das S. Common tumors and miscellaneous diseases of skin. A Concise Textbook of Surgery, $3^{\text {rd }}$ Edition; Calcutta, 2001, pp 82-124

5. Rydholm A, Berg NO. Size, site and clinical incidence of lipoma. Factors in the differential diagnosis of lipoma and sarcoma. Acta Orthop Scand. 1983 Dec;54(6):929-34. 